\title{
Failure of rapid diagnostic tests in Plasmodium falciparum malaria cases among travelers to the UK and Ireland: Identification and characterisation of the parasites
}

\author{
Debbie Nolder ${ }^{\mathrm{a}}$, Lindsay Stewart ${ }^{\mathrm{a}, \mathrm{b}}$, Julie Tucker ${ }^{\mathrm{a}}$, Amy Ibrahim $^{\mathrm{b}}$, Adam Gray, \\ Tumena Corrah ${ }^{c}$, Carmel Gallagher ${ }^{c}$, Laurence John ${ }^{c}$, Edel O’Brien ${ }^{\mathrm{d}}$, Dinesh Aggarwal ${ }^{\mathrm{e}}$, \\ Ernest Diez Benavente $^{\mathrm{b}}$, Donelly van Schalkwyk ${ }^{\mathrm{a}, \mathrm{b}}$, Gisela Henriques ${ }^{\mathrm{b}}$, \\ Nuno Sepúlveda ${ }^{\mathrm{b}, \mathrm{f}}$, Susana Campino $^{\mathrm{b}}$, Peter Chiodini ${ }^{\mathrm{a}, \mathrm{e}}$, Colin Sutherland ${ }^{\mathrm{a}, \mathrm{b}}$, \\ Khalid B. Beshir ${ }^{\mathrm{b}, *}$ \\ a PHE Malaria Reference Laboratory, London School of Hygiene and Tropical Medicine, Keppel Street, London WC1E 7HT, UK \\ bepartment of Infection Biology, Faculty of Infectious and Tropical Diseases, London School of Hygiene and Tropical Medicine, Keppel Street, London WC1E \\ 7HT, UK \\ c Department of Infectious Diseases, Northwick Park Hospital, London North West University Healthcare NHS Trust, London HA1 3UJ, UK \\ d Haematology Lab, University Hospital Limerick, Ireland \\ e Department of Clinical Parasitology, Hospital for Tropical Diseases, University College London Hospitals NHS Foundation Trust, London, UK \\ ${ }^{\mathrm{f}}$ CEAUL - Centro de Estatística e Aplicações da Universidade de Lisboa, Lisbon, Portugal
}

\section{A R T I C L E I N F O}

\section{Article history:}

Received 18 March 2021

Received in revised form 5 May 2021

Accepted 7 May 2021

\section{Keywords:}

RDT

Malaria

pfhrp2

pfhrp3

Deletion

Plasmodium

\begin{abstract}
A B S T R A C T
Objectives: Our objective was to systematically investigate false-negative histidine-rich protein 2 rapid diagnostic tests (HRP2-RDT) in imported Plasmodium falciparum malaria cases from travelers to the UK and the Republic of Ireland (RoI).

Methods: Five imported malaria cases in travellers returning to the UK and RoI from East Africa were reported to the PHE Malaria Reference Laboratory as negative according to histidine-rich protein (HRP2)RDT. The cases were systematically investigated using microscopic, RDT, molecular, genomic, and in in vitro approaches.

Results: In each case, HRP2-RDT was negative, whereas microscopy confirmed the presence of $P$. falciparum. Further analysis revealed that the genes encoding HRP2 and HRP3 were deleted in three of the five cases. Whole-genome sequencing in one of these isolates confirmed deletions in $P$. falciparum chromosomes 8 and 13. Our study produced evidence that the fourth case, which had high parasitemia at clinical presentation, was a rare example of antigen saturation ('prozone-like effect'), leading to a false negative in the HRP2-RDT, while the fifth case was due to low parasitemia.

Conclusions: False-negative HRP2-RDT results with $P$. falciparum are concerning. Our findings emphasise the necessity of supporting the interpretation of RDT results with microscopy, in conjunction with clinical observations, and sets out a systematic approach to identifying parasites carrying pfhrp2 and pfhrp 3 deletions.
\end{abstract}

(c) 2021 The Author(s). Published by Elsevier Ltd on behalf of International Society for Infectious Diseases. This is an open access article under the CC BY license (http://creativecommons.org/licenses/by/4.0/).

\section{Introduction}

Malaria rapid diagnostic tests (RDTs) are point-of-care immunochromatographic lateral flow assays which detect malaria

\footnotetext{
* Corresponding author at: Department of Infection Biology, Faculty of Infectious and Tropical Diseases, London School of Hygiene and Tropical Medicine, Keppel Street, London WC1E 7HT, UK.

E-mail address: khalid.beshir@lshtm.ac.uk (K.B. Beshir).
}

antigens in blood from patients with suspected malaria. RDTs play a key role in the diagnosis and appropriate timely treatment of malaria throughout malaria-endemic regions (Bruxvoort et al., 2017). Formats detecting Plasmodium falciparum histidine-rich proteins (HRP2, HRP3) are the most widely used RDTs in endemic countries. In non-endemic countries, these are typically used for initial diagnostic screening and/or to confirm the findings of blood film microscopy, which is considered the gold-standard test. The availability of reference laboratory confirmatory diagnosis by microscopy or PCR, in addition to RDT, is strongly recommended 
(Askling et al., 2012; Houze et al., 2013). This is because RDT detection of $P$. falciparum has lower sensitivity than other methods, and the false-negative rate resulting from this is exacerbated by the emergence of $P$. falciparum parasites that do not express both HRP2 and HRP3 antigens (Berhane et al., 2018; Gamboa et al., 2010). This phenomenon has become a malaria public health concern and threatens malaria control and case management efforts in endemic and non-endemic countries alike (Berhane et al., 2018; Beshir et al., 2017; Gamboa et al., 2010; Grignard et al., 2020; Watson et al., 2017).

$P$. falciparum parasites that fail to express the histidine-rich antigens are known to carry deletions of the pfhrp2 locus on chromosome 8 (gene ID: PF3D7_0831800) and/or the pfhrp3 locus on chromosome 13 (gene ID: PF3D7_1372200). Parasites with one or both loci deleted have been identified in the Americas, Africa, and some parts of Asia (Thomson et al., 2020). In Peru and Eritrea, HRP2-based RDTs are no longer deployed due to the high prevalence of HRP-deleted parasites (Berhane et al., 2018; Gamboa et al., 2010). While the global prevalence of parasites with the deletion remains low, and appears to be concentrated in certain populations, there is a growing recognition by WHO that these represent a potential threat to rapid diagnosis of falciparum malaria, a cornerstone of global control strategies (Gatton et al., 2017; Thomson et al., 2020).

The exact biological function of HRP2 is still unclear, but it has been linked with hemozoin formation, a process that converts a toxic, free heme into non-toxic, insoluble hemozoin during hemoglobin digestion (Sullivan et al., 1996). HRP2 has also been reported to be involved in cytoadherence to endothelial cells of postcapillary venules by erythrocytes infected with mature parasite stages. In a mouse model of $P$. falciparum infection, HRP2 is reported to be a virulence factor that may contribute to cerebral malaria (Pal et al., 2016). It is unknown whether $p f h r p 2 / 3$ deletion alters parasite fitness or disease severity in vivo. However, the analysis of genetic crosses between different laboratory strains has not revealed any deviation of Mendelian expectations for segregation of pfhrp2 deletions in the progeny (Sepulveda et al., 2018).

We have previously identified pfhrp2/pfhrp3-deleted parasites in malaria cases imported to the UK from Ethiopia, Kenya, Tanzania, Somalia, South Sudan, and Sudan (Grignard et al., 2020). In the present study we report clinical, parasitological, molecular, and genomic details of a series of five $P$. falciparumpositive cases presenting with false-negative HRP2-RDT results in the UK and the Republic of Ireland (RoI) between 2019 and 2021. We also report the successful culture adaptation of one pfhrp2/3deleted parasite isolate of Sudanese origin.

\section{Materials and methods}

Detection of malaria parasites in blood samples

Blood samples and fixed blood films from suspected or confirmed malaria patients are submitted by laboratories to the PHE Malaria Reference Laboratory (MRL) at the London School of Hygiene and Tropical Medicine (LSHTM) for confirmatory diagnosis. Films are examined by microscopy, as previously described (Nolder et al., 2013), and molecular analyses performed if microscopy is inconclusive and/or if there is any discrepancy between MRL and the sending laboratory diagnostic test results. RDTs are not used routinely, and are only deployed (according to manufacturer's instructions) when MRL microscopy results indicate a discrepancy with the sending laboratory's RDT findings.

Deletions and the presence of genetic polymorphisms located in $p$ fhrp 2 and $p f h r p 3$ are suspected when P. falciparum parasites are identified by microscopy or when $P$. falciparum DNA is confirmed using molecular analysis, and the sending laboratory has made an incorrect diagnosis based on RDT findings alone, such as reporting a non-falciparum malaria infection as the result.

\section{Molecular detection and speciation}

Molecular species diagnosis in the MRL combines nested PCR with species-specific $18 \mathrm{~S}$ ribosomal gene amplicons identified using gel electrophoresis (adapted from Calderaro et al., 2007; Padley et al., 2003; Snounou et al., 1993), and a probe-based qPCR method that discriminates Plasmodium species (adapted from Shokoples et al., 2009).

\section{Identification of pfhrp2/pfhrp3 deletion mutants}

Where $p$ fhrp $2 / 3$ deletions/mutations are suspected by the MRL, a panel of RDTs that target either pfhrp2/3 or PfLDH antigen are used to investigate. Available tests include: Carestart ${ }^{\mathrm{TM}}$ Malaria Rapydtest $^{\mathbb{B}}$ (HRP2/pLDH combo Pf/Pan-genus, Apacor); BinaxNOW Malaria (Pf HRP2/pan-genus aldolase, Alere) and OptiMAL-IT (Pf LDH/pan-genus LDH, Bio-Rad).

DNA was purified by a robotic DNA extraction system (Qiagen) following the manufacturer's instruction, as previously described (Robinson et al., 2019). Molecular testing for gene deletions was by assessment of parasite genomic DNA using a multiplex qPCR assay, as previously described (Grignard et al., 2020). Briefly, the method uses a multiplex assay targeting pfhrp2 and pfhrp3 as well as parasite reference and human house-keeping genes. Details of the genes and components of the qPCR have previously been described (Beshir et al., 2020; Grignard et al., 2020).

\section{Genomic analysis of pfhrp2 and pfhrp3 loci}

To confirm the pfhrp2/3 deletion and determine the extent of chromosome deletions, whole-genome sequencing (WGS) was performed using a previously described selected whole genome amplification (sWGA) method (Ibrahim et al., 2020; Oyola et al., 2016). The method selectively amplifies parasite DNA using short oligonucleotide probes of 8-12 mers as primers and enriches parasite DNA to generate whole-genome sequencing data from lowparasitemia samples. Details of the SWAG method, purification of the amplified samples, and library preparation of purified samples have previously been described (Ibrahim et al., 2020). All sequencing reactions were performed using paired $(2 \times) 150$ bp reads.

WGS sequence data analysis and adaptation of parasites to in vitro culture

The methods for whole-genome sequence data analysis and adaptation of $P$. falciparum isolates to in vitro culture are detailed in the supplementary material.

\section{Ethical approval}

Ethical approval for the samples from MRL patients was obtained from the NHS England Research Ethics Committee (18/LO/0738). Ethical approval for the laboratory work involving MRL samples was obtained from LSHTM Ethical Review Committee (\#14710).

\section{Results}

Patients

Patient 1 (sample S105)

In March 2019, a 72-year-old Sudanese female presented to the emergency department at a hospital in England with a 1-day 
history of symptoms suggestive of malaria. The patient had recently returned from visiting family in Sudan.

Blood films examined by the sending laboratory suggested malaria parasites which, following testing by Carestart RDT, were found to be positive for LDH antigen (pan-genus) only. The sample was referred to the MRL at LSHTM for confirmation of $P$. vivax.

\section{Patient 2 (sample S160)}

In September 2019, a 17-year-old British male of Somali heritage presented to the emergency department at a hospital in London with a 10-day history of symptoms suggestive of malaria, having returned from a 2-year stay in Uganda the day before clinical presentation. The patient had started feeling unwell in Uganda, presented to hospital, and been treated with antibiotics. He reported not receiving antimalarial treatment because his malaria diagnostic test was negative.

A provisional diagnosis of $P$. vivax was made after testing by Binax RDT, which was found to be positive for aldolase antigen (pan-genus) only. However, following microscopy, this diagnosis was revised to severe $P$. falciparum with $>20 \%$ parasitemia, and the patient was admitted to the intensive care unit, where a course of appropriate treatment was started. The hospital laboratory performed a Carestart RDT, which, despite the presence of microscopy-confirmed $P$. falciparum hyperparasitemia, generated only a weak positive signal for PfHRP2, but was positive for panLDH antigen. Blood films and an aliquot of EDTA blood were referred to the MRL for confirmation of species and investigation of inconsistency in RDT results. Biochemical data indicated that the patient had multiple markers of severe malaria (data not shown).

Patient 3 (sample S194)

In January 2020, a 28-year-old male returned to the UK from a 1 -week trip to Ethiopia to visit friends and family. He presented to the emergency department at a hospital in London 3 days after arrival, reporting a 5-day history of symptoms consistent with malaria. Scanty trophozoites of $P$. falciparum were reported despite a negative result using Binax RDT. The sample was sent to the Hospital for Tropical Diseases (HTD) in London for confirmation, because it operates a 24 -h out-of-hours malaria diagnosis service. The sample was initially reported as $P$. malariae following on-call microscopy together with a Carestart RDT result negative for the $P$. falciparum HRP2/3 antigens, but subsequent examination of the blood-film by HTD microscopists on the next working day reverted the previous diagnosis to P. falciparum infection and suspected false-negative RDT. The sample was sent to the MRL for molecular confirmation.

\section{Patient 4 (sample S204)}

In March 2020, a 55-year-old male returned to the RoI following a 3-week trip to Sudan, where he had been visiting friends and family. He reported presenting for medical care in Sudan, with symptoms suggestive of malaria, 6 days prior to his return to RoI.
Tests for malaria were reported to be negative. The patient then presented to the emergency department at a hospital in the RoI 3 weeks after arrival, a delay likely to have been caused by the rapidly emerging SARS-CoV-2 pandemic. Malaria parasites were seen on blood films and a Carestart RDT was positive for pLDH (pan-genus) antigen only, indicating a non-falciparum infection.

Samples from the patient were received by the MRL for confirmation; $P$. falciparum was diagnosed by microscopy and confirmed by molecular tests. RDTs were performed, confirming the sending laboratory's findings that the sample was negative for the HRP2/3 antigens. The sample was received within $72 \mathrm{~h}$ of being taken from the patient, and an aliquot was successfully established in long-term culture, designated as the parasite line HL2004.

Patient 5 (sample S227)

In January 2021, a 51-year-old female returned to the UK from 6-week stay in Zambia where she has been visiting family. The patient presented to a hospital in England in February and was diagnosed microscopically with malaria, species unknown, with scant parasites seen. A Carestart RDT was negative. Slides and an EDTA blood sample were sent to the MRL for confirmation and species diagnosis.

P. falciparum was diagnosed in the MRL by microscopy: early and late trophozoites with Maurer's clefts were seen. The sample was tested with Carestart, OptiMAL, and Binax RDTs and all three were negative for Plasmodium antigens, while Alethia ${ }^{\mathrm{TM}}$ Malaria LAMP (Meridian Bioscience) was positive. The qPCR indicated that the infection below $200 \mathrm{p} / \mu \mathrm{L}$. An aliquot was successfully established in long-term culture, designated as the parasite line HL2005.

\section{Diagnostic test results}

Diagnostic test results and investigations for the five blood samples are summarized in Tables 1 and 2. In each case, despite all five infections being confirmed as P. falciparum, inconsistencies in RDT results reported by the sending laboratory were also found by the MRL. These included weak or negative results obtained for HRP2/3 antigens detection by Carestart or Binax test kits, despite positive results for the optimal PfLDH antigen. We therefore suspected possible deletions of the respective genes in all five cases, and proceeded to assess the genomic status at both the pfhrp2 and pfhrp3 loci using multiplex qPCR.

\section{Confirmation of pfhrp2/3 deletion using multiplex $q P C R$}

Our one-step quadruplex qPCR assay amplifies three parasite genes ( $p f h r p 2, p f h r p 3$, and pfldh) and a human gene (HumTuBB) in a single reaction. All samples with suspected pfhrp2/3 deletions showed good amplification of the pfldh target gene (Figure 1), confirming the presence of good-quality parasite DNA in all samples. Three samples (S105, S160, and S204) were negative for

Table 1

Summary of investigations for blood samples from five malaria patients referred to the MRL following inconsistent RDT results.

\begin{tabular}{|c|c|c|c|c|c|c|c|}
\hline Sample reference & Travel history & $\begin{array}{l}\text { Carestart } \\
\text { Pf HRP }\end{array}$ & $\begin{array}{l}\text { Binax } \\
\text { Pf HRP }\end{array}$ & $\begin{array}{l}\text { Optimal } \\
\text { Pf LDH }\end{array}$ & $\begin{array}{l}\text { Carestart } \\
\text { Pan-genus LDH }\end{array}$ & $\begin{array}{l}\text { Binax } \\
\text { Pan-genus aldolase }\end{array}$ & $\begin{array}{l}\text { Optimal } \\
\text { Pan-genus LDH }\end{array}$ \\
\hline S105 & Sudan & $N E G$ & $N E G$ & POS & POS & POS & POS \\
\hline S160 & Uganda & WEAK & NEG/WEAK & POS & POS & POS & POS \\
\hline S194 & Ethiopia & $N E G$ & $N E G$ & POS & POS & POS & POS \\
\hline S204 & Sudan & $N E G$ & $N E G$ & POS & POS & POS & POS \\
\hline S227 & Zambia & $N E G$ & $N E G$ & $N E G$ & $N E G$ & $N E G$ & NEG \\
\hline
\end{tabular}

Pf: P. falciparum-specific.

LDH: parasite lactate dehydrogenase detectable by specific monoclonal antibodies (MAb) in the RDT.

Pan-genus: where RDT deploys an MAb that detects antigens from all members of the genus PlasmodiumE. 
Table 2

Microscopy, qPCR and parasite culture results for blood samples from five malaria patients referred to the MRL.

\begin{tabular}{|c|c|c|c|c|c|}
\hline Sample reference & Travel history & Initial diagnosis & Microscopy & P. falciparum DNA detected? & Culture established? \\
\hline S105 & Sudan & P. vivax & $\begin{array}{l}P . \text { falciparum trophozoites } \\
\mathrm{P} \sim 0.02 \%\end{array}$ & $\begin{array}{l}\text { P. falciparum DNA detected } \\
\text { Ct } 20.08\end{array}$ & No \\
\hline S160 & Uganda & P. vivax/P. falciparum & $\begin{array}{l}P . \text { falciparum trophozoites } \\
P \sim 24.3 \%\end{array}$ & Not done & No \\
\hline S194 & Ethiopia & P. falciparum/P. malariae & No films received & $\begin{array}{l}\text { P. falciparum DNA detected } \\
\text { Ct } 24.71\end{array}$ & No \\
\hline S204 & Sudan & Malaria parasites seen; unspecified & $\begin{array}{l}\text { P. falciparum, all stages } \\
\mathrm{P} \sim 0.1 \%\end{array}$ & $\begin{array}{l}\text { P. falciparum DNA detected } \\
\text { Ct } 22.88\end{array}$ & Yes (HL2004) \\
\hline S227 & Zambia & Malaria parasites seen; unspecified & $\begin{array}{l}P . \text { falciparum, late trophozoites } \\
P \sim 0.02 \%\end{array}$ & $\begin{array}{l}\text { P. falciparum DNA detected } \\
\text { Ct } 25.32\end{array}$ & Yes (HL2005) \\
\hline
\end{tabular}

Ct: cycle threshold - amplification cycle at which threshold for positivity is crossed by fluorescent signal in qPCR assays. Mean Ct values are given for two replicates.

pfhrp2 and pfhrp3 genes, as shown by the absence of amplification signal in both gene channels. Surprisingly, two samples (S160 and S227) showed amplification signals with Ct values of 22.0 and 28.6, respectively, for each of the genes, confirming the presence of both pfhrp2 and pfhrp3 (Figure 1, second row). The presence of amplification signals for both the parasite and human internal control genes indicated successful DNA extraction and PCR amplification in each case, and positive controls showed the expected amplification signal for all targets. The successfully culture-adapted samples (S204 and s227) were investigated for maintenance of the pfhrp $2 / 3$ deletion during the in vitro culture adaptation as isolates HL2004 and HL2005. Molecular analysis of the DNA extracted from HL2004 at days 21 and 28 following culture adaptation revealed that the sample generated no amplification signal for the pfhrp2/3 genes (Figure 2), while the HL2005 sample produced a good signal. The reference parasite gene $(p f l d h)$ produced a good amplification signal for both samples (data not shown). This confirmed that both culture-adapted isolates reflected the results seen in the original patient isolate (Figure 1, row 4).

\section{Further investigations for S160}

Given that qPCR analysis of the parasite DNA from S160 confirmed the presence of intact genes coding for both HRP antigens, sample dilution was performed to test the hypothesis

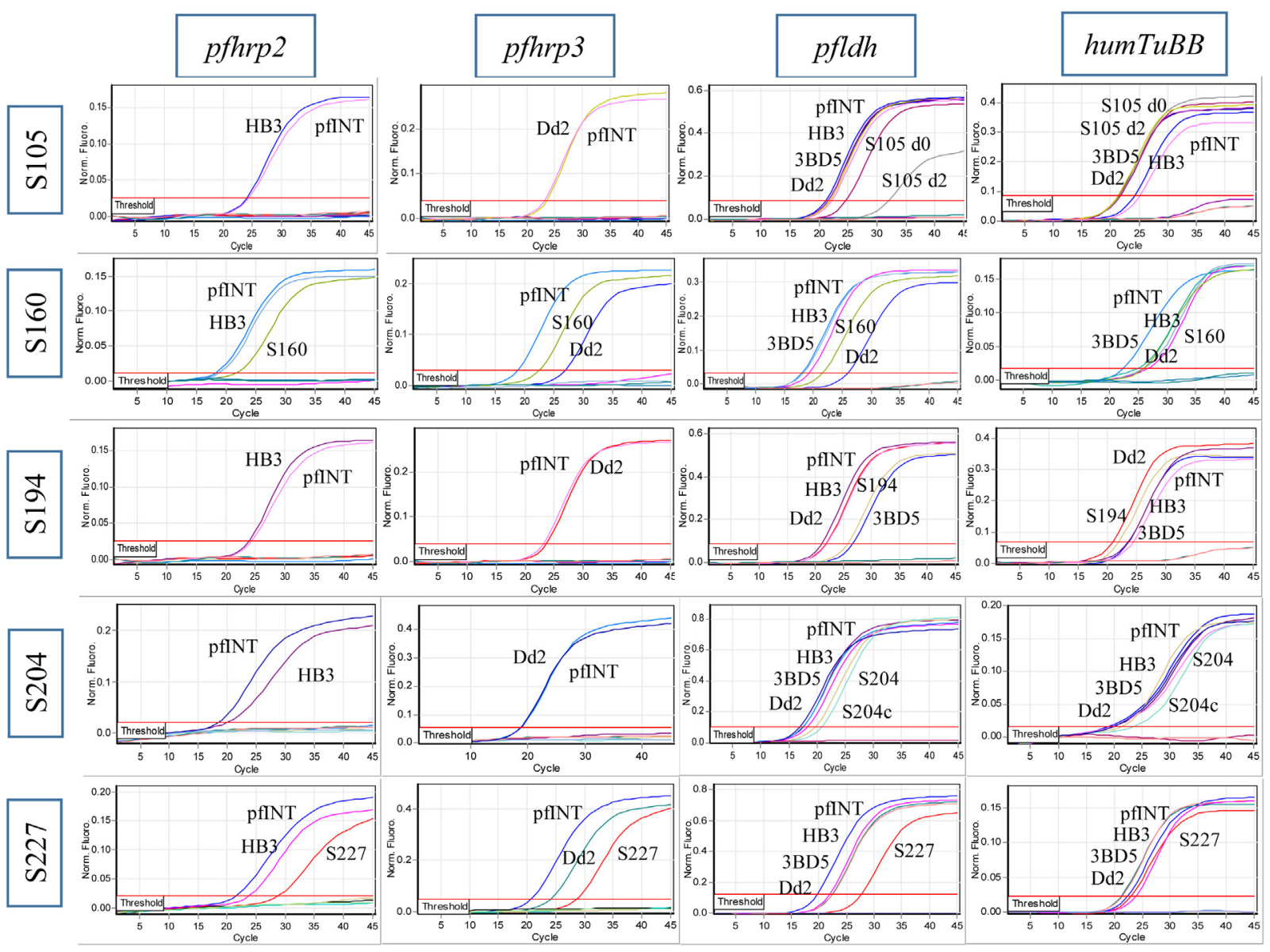

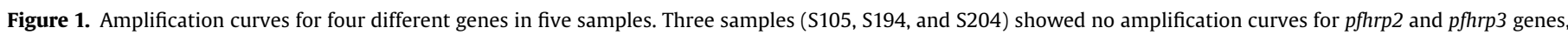

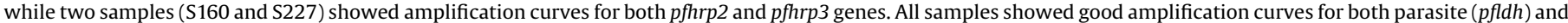

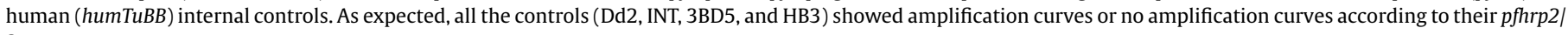
3 status. 
that antigen saturation (a 'prozone-like effect') may have accounted for the inconsistent RDT results for HRP antigen detection in this sample. The undiluted blood sample gave inconsistent results with HRP RDT, with a weak positive signal by Carestart ${ }^{\mathrm{TM}}$ Malaria Rapydtest ${ }^{\circledR}$ and a negative result by BinaxNOW. The original EDTA sample was diluted 1:10 in uninfected human blood. This dilution tested positive with both RDT formats, confirming the presence of both PfLDH and PfHRP antigens (data not shown).

Genomic analysis of pfhrp2 and pfhrp3 loci and P. falciparum isolate culture

Results for genomic analysis of pfhrp2/3 loci (inculding anlysis about Figure 3 ) and adaptation of $P$. falciparum isolates are presented in the Supplementary material.

\section{Discussion}

This study presents a systematic investigation of false-negative HRP2-RDT results in travelers to the UK and RoI with imported malaria due to P. falciparum infection. Clinical, molecular, genomic, and in vitro evidence is provided, confirming the deletion of pfhrp2/ 3 genes in parasites from three patients with false-negative results by HRP2-RDT. This is the first study to show pfhrp2/3 deletion in patient samples from UK travelers causing false-negative HRP2RDT results. The study also presents evidence that a false-negative RDT result obtained for a fourth patient was due to a prozone-like effect due to antigen saturation caused by high parasitemia, and for a fifth patient due to low parasitemia.

We have previously identified pfhrp $2 / 3$ deletions in a molecular survey of $P$. falciparum parasites imported into the UK (Grignard et al., 2020), but these were not linked to diagnostic test failure. In this study, we show the potentially serious impact of imported parasites with HRP deletions on RDT performance in the UK and RoI. With the increase in frequency of such $P$. falciparum parasites in endemic countries, over-reliance on HRP2-RDT diagnosis in the UK or RoI may delay treatment for sick individuals, with lifethreatening consequences. One patient in our series with falsenegative RDT results was admitted to intensive care unit with hyperparasitemia; he reported receiving no antimalarial treatment in Uganda prior to travel due to a negative malaria test. Early presentation, rapid diagnosis, and immediate treatment can together greatly reduce severe malaria risk, and are the basis of effective malaria management in non-endemic countries (Checkley et al., 2012).

The prozone effect due to high parasitemia has been previously demonstrated in laboratory studies of both clinical isolates and
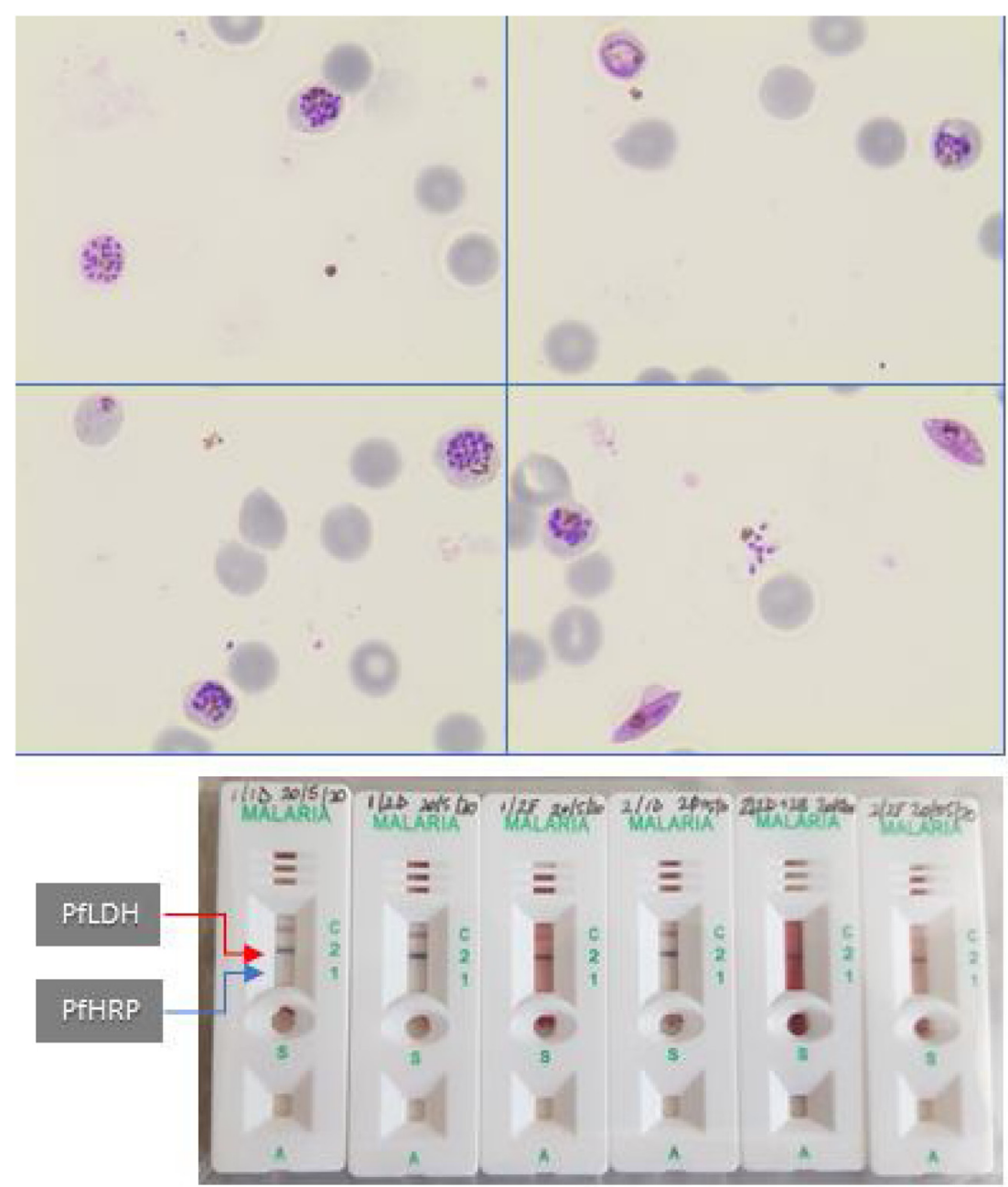

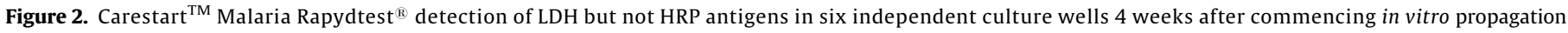

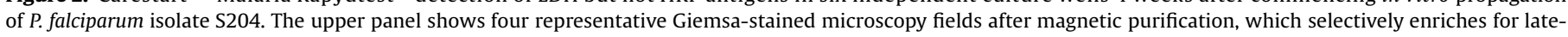

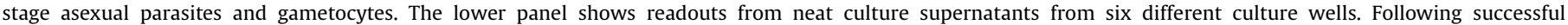

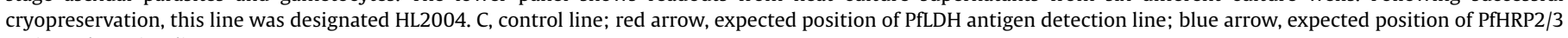
antigen detection line. 
laboratory cultures (Luchavez et al., 2011). Variation in RDT susceptibility between RDT products was observed, reflecting either the quality of the RDT manufacture or the high specificity of commercially produced proprietary monoclonal antibodies used on the test devices. Other researchers have reported prozone-like effects at parasitemia leves of 5.5-35\% (Gillet et al., 2009), and there is evidence that high concentrations of patient antibodies against HRP2/3 antigens may also contribute to the inhibition of RDT recognition of antigens (Ho et al., 2014). Thus pfhrp2/3 deletion is not the only factor that contributes to false HRP2-RDT negative results, and both factors should be considered when faced with HRP2-RDT negative results in patients with suspected malaria. Our findings also emphasize the need for ruling out low parasitemia as a possible factor for false-negative RDT results, as extensively demonstrated in our previous report (Beshir et al., 2017).

For the present study, a multiplex qPCR assay - recently validated as a one-step genotyping method (Grignard et al., 2020)
- was used to demonstrate that false-negative RDT results were caused by the deletion of $p f h r p 2 / 3$ in three patients. Our results confirmed that pfhrp2/3-deleted parasites are in circulation in Ethiopia and Sudan, as previously reported (Boush et al., 2020; Girma et al., 2019; Golassa et al., 2020; Mussa et al., 2019). Interestingly, a mathematical modelling exercise (Watson et al., 2017) predicted the insurgence of such parasites in African countries with low endemicity, such as Sudan, Somalia, or Ethiopia, which were the travel origins of the patients we analysed. However, it is unclear how extensively these parasites circulate in these countries. At the genomic level, the S105 clinical sample from Sudan exhibited zero sequence coverage at the pfhrp2 and pfhrp3 loci, confirming deletion of the two genes and flanking regions. The deletion of a large proportion of loci surrounding pfhrp2 and pfhrp3 is similar to the regional loss observed in laboratory lines Dd2 and HB3 (Sepulveda et al., 2018), though the loss in Dd2 is smaller (14 kb) compared with the clinical sample described here $(\sim 50 \mathrm{~kb})$. The implication of the loss of genes
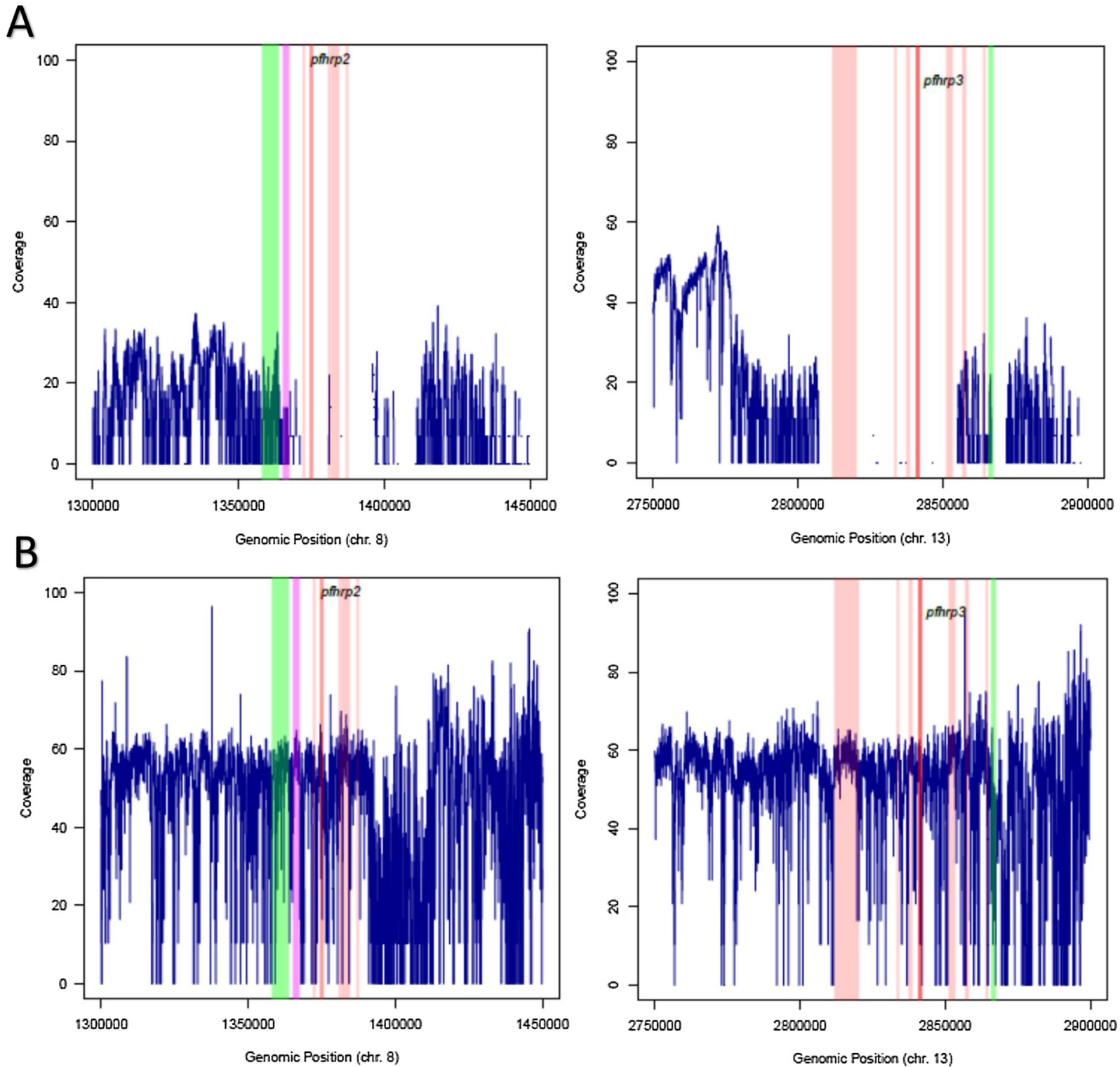

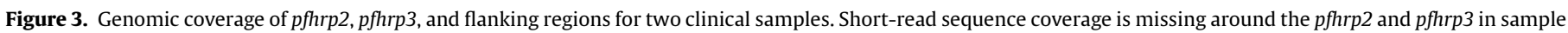

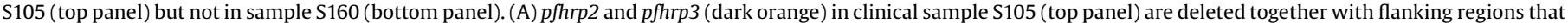

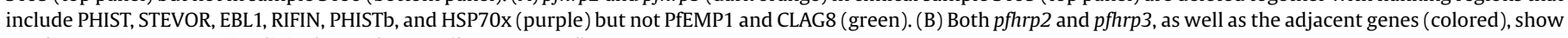
good genomic coverage in clinical sample S160 (bottom panel). 
adjacent to pfhrp2 and pfhrp3 is unknown. It is important to note that the S160 patient had signs and symptoms attributable to severe malaria, and it is not clear whether the presence of HRP2 and flanking regions contributed to the severity (Pal et al., 2016) or it was simply caused by high parasitemia (Kingston et al., 2017). Cytoadherence - a characteristic of cerebral malaria - is thought to be mediated by parasite proteins such as RIFINs, STEVORs, HSP70x, and pfEMP1 (Sherman et al., 2003), all of which are located in the vicinity of the pfhrp2 or pfhrp 3 loci. Whether the loss of $p$ fhrp $2 / 3$ or the flanking regions has any role in parasite fitness or virulence requires thorough investigation in future studies, including precision mapping of chromosome breakpoints in multiple parasites carrying HRP gene deletions.

Attempts to culture-adapt the clinical isolates was successful for one from a traveller returning to the RoI from Sudan, which lacked both HRP genes, and one from a UK traveller returning from Zambia, which harboured both genes. The growth of HRP-negative parasite line HL2004 was poor compared with other patientderived lines with intact pfhrp2/3 previously cultured in our laboratory (van Schalkwyk et al., 2013). It is not clear whether poor growth was due to a loss of fitness associated with the deletion of pfhrp $2 / 3$, the delay in establishing the culture, or any other technical factors. Disruption of pfhrp2 in the 3D7 laboratory line has been reported to have no effect on parasite growth (Yang et al., 2020); however, the pfhrp2 locus alone was disrupted by gene replacement in this study, and so any fitness impact due to loss of flanking genes was not examined. The exact biological function of HRP2 is still unclear, but it has been linked with hemozoin formation in several studies (Schneider and Marletta, 2005; Sullivan et al., 1996). The pfhrp2 knock-out 3D7 parasite line has been shown to transcriptionally downregulate enzymes required for heme metabolism in the food vacuole, but to upregulate transcripts for proteins required for heme biosynthesis in the apicoplast, potentially maintaining a relatively unchanged heme level in the parasite (Yang et al., 2020). This might indicate that pfhrp2 deletion can impact parasite survival in the mosquito and liver stages, as de novo heme biosynthesis in the apicoplast has been reported to be essential in the mosquito (Ke et al., 2014) and liver stages (Nagaraj et al., 2013). Clonal expansion of pfhrp2deleted parasites has been observed in Eritrea and Ethiopia (Berhane et al., 2018; Girma et al., 2019; Golassa et al., 2020), but it remains unclear whether the parasites with pfhrp2 deletion have a fitness advantage or deficit compared with the wild type. In areas where RDT-testing and treatment is a policy, the pfhrp2-deleted parasites would evade HRP2-RDT detection and would therefore not be exposed to antimalarial onslaught. This potentially gives pfhrp2-deleted parasites survival and expansion advantages over the wild type (Watson et al., 2017). Mosquito transmission studies are now needed to understand the role of $p f h r p 2 / 3$ and flanking regions in the expansion of pfhrp2/3-deleted parasite populations in malaria-endemic countries. The gametocyte-producing property of HL2004 suggests that this isolate is ideal for such a study.

Our study had some limitations, including the small number of clinical samples in which the impact of pfhrp2/3 deletion on RDT performance in the UK and RoI was evaluated. Similarly, the precise role of high parasitemia on HRP2-RDT performance, which dramatically affected diagnostic results for one patient reported here, requires assessment in a larger patient series. Finally, the culture-adapted HRP-negative isolate will undoubtedly prove a useful research tool as well as a potential source of reference material for evaluating RDT performance against gene-deleted parasites, but may not provide a generalizable model of the fitness cost of pfhrp $2 / 3$ deletion. Isolation of additional culture-adapted HRP-deleted $P$. falciparum lines of clinical origin should be prioritised.
Our investigations enhance the existing evidence base, and support the continued use of RDT as a rapid and convenient screening tool for imported malaria, but one that should only be deployed when backed-up by high-quality microscopy and capacity for DNA-based molecular tests. Although RDT diagnostic failures remain rare among imported UK malaria cases, molecular surveillance of $p f h r p 2 / 3$ needs to be maintained so that the impact of imported P. falciparum with pfhrp $2 / 3$ deletion on RDT performance in the UK can be continuously monitored.

\section{Funding}

This work was supported by a Wellcome Institutional Strategic Support Fund granted to KBB $(204928 / Z / 16 / Z)$. DN is funded by the Public Health England Malaria Reference Laboratory. The Malaria Reference Laboratory is funded by Public Health England (research contract awarded to CJS). The funders did not have any role in study design, data collection, data analysis, interpretation, or writing of the report.

\section{Conflicts of interest}

KBB declares financial support from the World Health Organization. Other authors declare no conflicts of interest.

\section{Acknowledgements}

The authors would like to thank all the patients who participated in this study.

\section{Appendix A. Supplementary data}

Supplementary material related to this article can be found, in the online version, at doi:https://doi.org/10.1016/j. ijid.2021.05.008.

\section{References}

Askling HH, Bruneel F, Burchard G, Castelli F, Chiodini PL, Grobusch MP, et al. Management of imported malaria in Europe. Malar J 2012;11:328.

Berhane A, Anderson K, Mihreteab S, Gresty K, Rogier E, Mohamed S, et al. Major threat to malaria control programs by Plasmodium falciparum lacking histidinerich protein 2, Eritrea. Emerg Infect Dis 2018;24(3):462-70.

Beshir KB, Grignard L, Hajissa K, Mohammed A, Nurhussein AM, Ishengoma DS, et al. Emergence of undetectable malaria parasites: a threat under the radar amid the COVID-19 pandemic?. Am J Trop Med Hyg 2020;103(2):558-60.

Beshir KB, Sepulveda N, Bharmal J, Robinson A, Mwanguzi J, Busula AO, et al. Plasmodium falciparum parasites with histidine-rich protein 2 (pfhrp2) and pfhrp3 gene deletions in two endemic regions of Kenya. Sci Rep 2017;7 (1): 14718 .

Boush MA, Djibrine MA, Mussa A, Talib M, Maki A, Mohammed A, et al. Plasmodium falciparum isolate with histidine-rich protein 2 gene deletion from Nyala City, Western Sudan. Sci Rep 2020;10(1):12822.

Bruxvoort KJ, Leurent B, Chandler CIR, Ansah EK, Baiden F, Bjorkman A, et al. The impact of introducing malaria rapid diagnostic tests on fever case management: a synthesis of ten studies from the ACT Consortium. Am J Trop Med Hyg 2017;97 (4):1170-9.

Calderaro A, Piccolo G, Perandin F, Gorrini C, Peruzzi S, Zuelli C, et al. Genetic polymorphisms influence Plasmodium ovale PCR detection accuracy. J Clin Microbiol 2007;45(5):1624-7.

Checkley AM, Smith A, Smith V, Blaze M, Bradley D, Chiodini PL, et al. Risk factors for mortality from imported falciparum malaria in the United Kingdom over 20 years: an observational study. BMJ 2012;344:e2116.

Gamboa D, Ho M-F, Bendezu J, Torres K, Chiodini PL, Barnwell JW, et al. A large proportion of $P$. falciparum isolates in the Amazon Region of Peru lack pfhrp2 and pfhrp3: implications for malaria rapid diagnostic tests. PLoS One 2010;5(1): e8091.

Gatton ML, Dunn J, Chaudhry A, Ciketic S, Cunningham J, Cheng Q. Implications of parasites lacking Plasmodium falciparum histidine-rich protein 2 on malaria morbidity and control when rapid diagnostic tests are used for diagnosis. J Infect Dis 2017;215(7):1156-66.

Gillet P, Mori M, Van Esbroeck M, Van den Ende J, Jacobs J. Assessment of the prozone effect in malaria rapid diagnostic tests. Malar J 2009;8:271. 
Girma S, Cheaveau J, Mohon AN, Marasinghe D, Legese R, Balasingam N, et al. Prevalence and epidemiological characteristics of asymptomatic malaria based on ultrasensitive diagnostics: a cross-sectional study. Clin Infect Dis 2019;69 (6):1003-10.

Golassa L, Messele A, Amambua-Ngwa A, Swedberg G. High prevalence and extended deletions in Plasmodium falciparum hrp2/3 genomic loci in Ethiopia. PLoS One 2020;15(11):e0241807.

Grignard L, Nolder D, Sepulveda N, Berhane A, Mihreteab S, Kaaya R, et al. A novel multiplex qPCR assay for detection of Plasmodium falciparum with histidine-rich protein 2 and 3 (pfhrp2 and pfhrp3) deletions in polyclonal infections. EBioMedicine 2020;55:102757.

Ho MF, Baker J, Lee N, Luchavez J, Ariey F, Nhem S, et al. Circulating antibodies against Plasmodium falciparum histidine-rich proteins 2 interfere with antigen detection by rapid diagnostic tests. Malar J 2014;13:480.

Houze S, Boutron I, Marmorat A, Dalichampt M, Choquet C, Poilane I, et al. Performance of rapid diagnostic tests for imported malaria in clinical practice: results of a national multicenter study. PLoS One 2013;8(9):e75486.

Ibrahim A, Diez Benavente E, Nolder D, Proux S, Higgins M, Muwanguzi J, et al. Selective whole genome amplification of Plasmodium malariae DNA from clinical samples reveals insights into population structure. Sci Rep 2020;10 (1):10832.

Ke H, Sigala PA, Miura K, Morrisey JM, Mather MW, Crowley JR, et al. The heme biosynthesis pathway is essential for Plasmodium falciparum development in mosquito stage but not in blood stages. J Biol Chem 2014;289(50):34827-37.

Kingston HW, Ghose A, Plewes K, Ishioka H, Leopold SJ, Maude RJ, et al. Disease severity and effective parasite multiplication rate in falciparum malaria. Open Forum Infect Dis 2017;4(4):ofx169.

Luchavez J, Baker J, Alcantara S, Belizario Jr. V, Cheng Q, McCarthy JS, et al. Laboratory demonstration of a prozone-like effect in HRP2-detecting malaria rapid diagnostic tests: implications for clinical management. Malar J 2011;10:286

Mussa A, Talib M, Mohamed Z, Hajissa K. Genetic diversity of Plasmodium falciparum histidine-rich protein 2 (PfHRP2) and its effect on the performance of PfHRP2based rapid diagnostic tests. BMC Res Notes 2019;12(1):334.

Nagaraj VA, Sundaram B, Varadarajan NM, Subramani PA, Kalappa DM, Ghosh SK, et al. Malaria parasite-synthesized heme is essential in the mosquito and liver stages and complements host heme in the blood stages of infection. PLoS Pathog 2013;9(8):e1003522.

Nolder D, Oguike MC, Maxwell-Scott H, Niyazi HA, Smith V, Chiodini PL, et al. An observational study of malaria in British travellers: Plasmodium ovale wallikeri and Plasmodium ovale curtisi differ significantly in the duration of latency. BMJ Open 2013;3(5).
Oyola SO, Ariani CV, Hamilton WL, Kekre M, Amenga-Etego LN, Ghansah A, et al Whole genome sequencing of Plasmodium falciparum from dried blood spots using selective whole genome amplification. Malar J 2016;15(1):597.

Padley D, Moody AH, Chiodini PL, Saldanha J. Use of a rapid, single-round, multiplex PCR to detect malarial parasites and identify the species present. Ann Trop Med Parasitol 2003;97(2):131-7.

Pal P, Daniels BP, Oskman A, Diamond MS, Klein RS, Goldberg DE. Plasmodium falciparum histidine-rich protein II compromises brain endothelial barriers and may promote cerebral malaria pathogenesis. mBio 2016;7(3).

Robinson A, Busula AO, Muwanguzi JK, Powers SJ, Masiga DK, Bousema T, et al. Molecular quantification of Plasmodium parasite density from the blood retained in used RDTs. Sci Rep 2019;9(1):5107.

Schneider EL, Marletta MA. Heme binding to the histidine-rich protein II from Plasmodium falciparum. Biochemistry 2005;44(3):979-86.

Sepulveda N, Phelan J, Diez-Benavente E, Campino S, Clark TG, Hopkins H, et al. Global analysis of Plasmodium falciparum histidine-rich protein-2 (pfhrp2) and pfhrp3 gene deletions using whole-genome sequencing data and meta-analysis. Infect Genet Evol 2018;62:211-9.

Sherman IW, Eda S, Winograd E. Cytoadherence and sequestration in Plasmodium falciparum: defining the ties that bind. Microbes Infect 2003;5 (10):897-909.

Shokoples SE, Ndao M, Kowalewska-Grochowska K, Yanow SK. Multiplexed realtime PCR assay for discrimination of Plasmodium species with improved sensitivity for mixed infections. J Clin Microbiol 2009;47(4):975-80.

Snounou G, Viriyakosol S, Zhu XP, Jarra W, Pinheiro L, do Rosario VE, et al. High sensitivity of detection of human malaria parasites by the use of nested polymerase chain reaction. Mol Biochem Parasitol 1993;61(2):315-20.

Sullivan Jr. DJ, Gluzman IY, Goldberg DE. Plasmodium hemozoin formation mediated by histidine-rich proteins. Science 1996;271(5246):219-22.

Thomson R, Parr JB, Cheng Q, Chenet S, Perkins M, Cunningham J. Prevalence of Plasmodium falciparum lacking histidine-rich proteins 2 and 3: a systematic review. Bull World Health Organ 2020;98(8):558-568F.

van Schalkwyk DA, Burrow R, Henriques G, Gadalla NB, Beshir KB, Hasford C, et al. Culture-adapted Plasmodium falciparum isolates from UK travellers: in vitro drug sensitivity, clonality and drug resistance markers. Malar J 2013:12:320.

Watson OJ, Slater HC, Verity R, Parr JB, Mwandagalirwa MK, Tshefu A, et al Modelling the drivers of the spread of Plasmodium falciparum hrp2 gene deletions in sub-Saharan Africa. eLife 2017;6:

Yang Y, Tang T, Feng B, Li S, Hou N, Ma X, et al. Disruption of Plasmodium falciparum histidine-rich protein 2 may affect haem metabolism in the blood stage. Parasit Vectors 2020;13(1):611 